\title{
Conséquences des restrictions pondérales imposées par le sport chez l'enfant et l'adolescent
}

\author{
Nathalie Boisseau \\ LAPHAP, Faculté des Sciences du Sport, Poitiers, France
}

\author{
Mots-clés \\ Sport dans l'enfance et l'adolescence • Restriction pondérale • \\ Croissance, enfance $\cdot$ Maturité
}

\section{Résumé}

De nos jours, de jeunes sportifs de compétition entreprennent des programmes d'entraînement comportant un haut degré d'activité physique dès un âge précoce. Cet entraînement intensif (volume et intensité) augmente les dépenses énergétiques, qui doivent être compensées par une augmentation des apports nutritionnels. Les danseurs de corps de ballet, les patineurs artistiques, les gymnastes ou les compétiteurs de gymnastique rythmique commencent leur entraînement dès l'âge de 5-6 ans et, pour la plupart d'entre eux, consacrent plus de 20 à 30 heures par semaine à leur discipline. Dans ces activités, ainsi que dans celles où le poids joue un rôle (lutteurs, jockeys, judokas ou boxeurs), les jeunes sportifs de haut niveau décident délibérément de réduire leur consommation alimentaire afin de maintenir leur minceur physique prépubertaire ou de «faire le poids». Des apports adéquats en calories et en micronutriments sont essentiels chez l'enfant en croissance, et des restrictions caloriques et liquidiennes au cours de l'entraînement physique pendant l'enfance ou l'adolescence pourraient perturber les régulations métaboliques et hormonales intervenant dans la croissance, la maturité, la composition corporelle, les cycles menstruels et l'aptitude à la reproduction, et pourraient augmenter le risque de lésions telles que des fractures de contrainte. De plus, ces stratégies peuvent entraîner l'apparition de troubles alimentaires (anorexie mentale et/ou boulimie nerveuse), particulièrement chez les adolescentes, affectant I'image corporelle et l'estime de soi et favorisant des inadaptations sociales et affectives.

Copyright ๑ 2006 Nestec Ltd., Vevey/S. Karger AG, Basel

\section{Introduction}

Au cours de l'enfance et de l'adolescence, une croissance et un développement rapide augmentent les besoins en énergie et en micronutriments. Les besoins nutritionnels sont plus élevés chez les jeunes sportifs en raison de leur activité physique plus importante. Les restrictions alimentaires et liquidiennes sont conséquentes chez les jeunes sportifs de haut niveau, particulièrement chez ceux pratiquant un sport faisant intervenir l'esthétique ou le poids, et peuvent être potentiellement dangereuses au cours de la période de croissance. L'objectif de la présente analyse est de présenter les effets indésirables potentiels pour la santé des restrictions pondérales imposées par un sport au cours de l'enfance et l'adolescence.

\section{Restrictions alimentaires et liquidiennes dans des catégories spécifiques de sport}

\section{Energie, macro-et micronutriments}

Au cours de l'enfance et de l'adolescence, une croissance et un développement rapides influencent les besoins en énergie ainsi qu'en macro- et micronutriments.

\section{KARGER \\ Fax +41 613061234 E-Mail karger@karger.ch} www.karger.com (c) 2006 Nestec Ltd., Vevey/S. Karger AG, Basel 0250-9644/06/0642-0077\$23.50/0

Accessible en ligne à:

www.karger.com/anf
Dr. Nathalie Boisseau

LAPHAP, Faculté des Sciences du Sport

4 allée Jean-Monnet

FR-86000 Poitiers (France)

Tel. +33 549454 042, Fax +33 549453 396, E-Mail nathalie.boisseau@univ-poitiers.fr 


\section{Energie}

En raison d'une importante variabilité intra- et interindividuelle, il est relativement difficile de quantifier les besoins énergétiques estimés de l'enfant ou de l'adolescent sportif ou très actif. Les calculs du Food and Nutrition Board estimant les besoins énergétiques reposent sur des équations incluant l'âge, la taille et le poids corporel du sujet ainsi qu'une classification physique en mode de vie sédentaire, modérément actif, actif et très actif [1]. Chez les enfants de 9-13 ans, les besoins énergétiques vont de $1415 \mathrm{kcal} / j o u r$ chez une fille de 9 ans sédentaire à $3038 \mathrm{kcal} /$ jour chez un garçon de 13 ans très actif. Chez les adolescents de 14-18 ans, les besoins énergétiques vont de $1718 \mathrm{kcal} /$ jour pour la jeune fille sédentaire de 14 ans à $3804 \mathrm{kcal} /$ jour pour l'adolescent très actif de 18 ans. De ce fait, les dépenses énergétiques totales des enfants et adolescents sont liées au metabolisme de base, à la croissance et au degré d'activité physique. Par exemple, chez les gymnastes de haut niveau, l'entraînement physique augmente la demande calorique et induit une consommation supplémentaire de 400-700 kcal d'énergie par jour [2, 3].

\section{Apport énergétique dans les sports impliquant une restriction pondérale}

Afin de demeurer mince et de conserver un aspect physique juvénile, de nombreux athlètes de haut niveau (gymnastique, gymnastique rythmique, patinage artistique, danse) sont convaincus qu'ils doivent suivre un régime alimentaire. Les pratiquants des sports comportant une classification en fonction du poids (lutteurs, judokas ou boxeurs) appliquent fréquemment des restrictions alimentaires et liquidiennes pendant toute la saison des compétitions afin de concourir dans une catégorie de poids une à trois classes inférieure à celle de leur poids normal [4], pensant ainsi acquérir un avantage sur des adversaires plus petits. De ce fait, des cycles réitérés de perte et de reprise de poids sont habituels, et les apports énergétiques sont régulièrement perturbés $[5,6]$. Ces pertes et reprises de poids ont été associées à une réduction des besoins énergétiques dus à une diminution du métabolisme chez les jeunes sportifs [7].

Quelques études, bien que limitées, ont montré que les apports énergétiques sont théoriquement insuffisants pour soutenir la croissance et un entraînement intensif pendant toute la durée du programme d'entraînement (gymnastes, gymnastes rythmiques, danseurs de ballet, etc.) ou de la saison des compétitions chez les sportifs soumis à des catégories de poids [8-20].

\section{Protéines}

Des apports protéiques suffisants sont nécessaires à la fourniture d'acides aminés essentiels qui soutiennent la croissance, et particulièrement le maintien et le développement de la masse maigre. De fait, le métabolisme protéique est important pour la croissance et le remodelage des tissus maigres. Un entraînement physique intensif et régulier augmente les besoins protéiques quotidiens des sportifs adultes $[1,21]$. Les apports protéiques recommandés chez les enfants et adolescents sédentaires ou modérément actifs (âgés de 9 à 18 ans) vont de 0,73 à $0,85 \mathrm{~g} / \mathrm{kg}$ [1] ou de 0,80 à $1,0 \mathrm{~g} / \mathrm{kg}$ de masse corporelle $[22,23]$. Cette recommandation ne concerne toutefois pas les enfants actifs ou très actifs. En fait, aucune donnée n'est disponible quant aux besoins protéiques des jeunes sportifs. Dans la plupart des cas, les jeunes concurrents augmentent spontanément leurs apports alimentaires en énergie et satisfont de façon naturelle leurs besoins protéiques. Dans les sports aboutissant à des comportements de restriction alimentaire, le métabolisme protéique pourrait être perturbé et, de ce fait, entraîner une altération de la croissance et/ou de la masse non grasse totale.

Dans le cas des gymnastes féminines ou des ballerines, même si des évaluations nutritionnelles indiquent que les apports énergétiques sont inférieurs aux apports nutritionnels habituellement conseillés [1] chez un grand nombre de ces jeunes filles pour leur âge et leur degré d'activité physique [17,24-27], les apports protéiques spontanés paraissent être supérieurs à $0,8-1,0 \mathrm{~g} / \mathrm{kg} /$ jour et atteindre $1,3-1,8 \mathrm{~g} / \mathrm{kg} /$ jour la plupart du temps. De fait, comme diverses études nutritionnelles l'ont indiqué, ces sportifs consomment des protéines en excès aux dépends des lipides et des glucides [27]. De plus, une récente étude utilisant l'isotope stable ${ }^{15} \mathrm{~N}$-glycine a indiqué que l'entraînement n'affectait pas le renouvellement des protéines chez de jeunes gymnastes féminines en période prépubertaire ou au début de la puberté [17].

L'attitude concernant les jeunes lutteurs ou judokas de haut niveau est très différente. Ainsi, différentes publications ont montré que le statut protéique nutritionnel pouvait être altéré au cours de la période de perte de poids [28], ce qui pourrait induire un bilan azoté temporairement négatif pouvant aboutir à des perturbations de la croissance et à des lésions musculaires. Friedlander et coll. [29] ont récemment indiqué qu'une restriction calorique d'une durée de 3 semaines altérait le métabolisme protéique (renouvellement et oxydation de la leucine) chez de jeunes hommes de poids normal.

\section{Glucides}

Les apports nutritionnels de référence concernant les glucides reposent sur la quantité nécessaire à l'apport de glucose à des tissus essentiels (tels que le cerveau) et ne prennent pas en compte la nécessité du remplacement du glycogène musculaire ou hépatique. Chez l'adulte, les performances atteintes lors d'efforts prolongés ou répétés de haute intensité sont liées aux stocks totaux de glycogène, car les glucides sont les substrats énergétiques limitants pour le maintien du taux de travail $[30,31]$. Chez les enfants prépubertaires, la capacité glycolytique paraît être moins efficiente que chez les adolescents ou les adultes, ce qui induit un 
accroissement de l'utilisation des graisses au cours d'effort prolongé chez les jeunes sportifs [32]. Il reste encore à déterminer si les jeunes sportifs bénéficient ou non d'apports glucidiques élevés. Les apports glucidiques recommandés sont de 50 à $60 \%$ de la consommation énergétique quotidienne totale, avec seulement $10 \%$ de sucres simples [33].

Les gymnastes et les danseurs de ballet sont persuadés que les glucides sont des aliments riches en calories. Bien que la consommation moyenne de glucides chez les gymnastes, les ballerines et les patineurs artistiques, par exemple, soit de $\pm 50 \%$ des apports énergétiques totaux, ces apports se composent essentiellement de sucres simples et non d'hydrates de carbone complexes [8, 17, 24, 27, 34]. Ces enquêtes nutritionnelles indiquent que la préférence pour le goût sucré n'est pas bien adaptée à l'augmentation des stocks de glycogène musculaire et hépatique. Dans les sports comportant des catégories de poids, les restrictions alimentaires pourraient également induire une diminution significative de la teneur musculaire et hépatique en glycogène immédiatement avant la compétition [5].

\section{Lipides}

Il n'existe aucun apport quotidien adéquat ou recommandé pour les lipides totaux, mais il existe des consommations adéquates concernant deux acides gras essentiels, les acides linoléique et linolénique ( $\mathrm{n}-3$ et n-6; tableau 1 ).

Chez l'enfant et l'adolescent, il est recommandé que 30\% des apports énergétiques totaux proviennent de lipides [1, 33]. Bien que, lors d'efforts prolongés, l'enfant utilise proportionnellement plus de lipides comme source d'énergie que l'adulte, aucune donnée ne suggère qu'il puisse nécessiter des apports lipidiques plus importants.

Concernant l'objectif de contrôle du poids ou de restriction pondérale, il est habituel de suggérer une diminution de la consommation de lipides. Un diminution de l'apport énergétique total par réduction de lipides peut s'avérer une stratégie dangereuse chez un enfant ou un adolescent sportif. De fait, une quantité minimale de graisse corporelle est nécessaire pour assurer les adaptations métaboliques et hormonales. La proportion recommandée pour le maintien de la santé et de la condition physique est de 7\% chez les garçons et de 14\% chez les filles [35]. La réduction, ou même l'éviction, des produits riches en lipides est une aberration aboutissant à des carences en graisses monoinsaturées et polyinsaturées. De plus, des produits riches en lipides tels que les produits laitiers et la viande rouge contiennent diverses substances (protéines de haute qualité, calcium, magnésium, fer, zinc, chrome, vitamine $B_{12}$, vitamines liposolubles, etc.) nécessaires à l'optimisation de la croissance. Toutes les enquêtes nutritionnelles sur les sports à critères esthétiques, dont la danse, le patinage artistique, la gymnastique et la gymnastique rythmique, indiquent que les apports lipidiques sont insuffisants $[8,11,17,24,27]$. Dans les
Tableau 1. Apports adéquats en acides linoléique et linolénique

\begin{tabular}{lll}
\hline Age & \multicolumn{2}{l}{ Acide gras, g/jour } \\
\cline { 2 - 3 } & acide linoléique & acide linolénique \\
\hline $9-13$ ans & & \\
$\quad$ Garçons & 12 & 1,2 \\
Filles & 10 & 1,0 \\
14-18 ans & 16 & 1,6 \\
Adolescents & 11 & 1,1 \\
$\quad$ Adolescentes & & \\
\hline
\end{tabular}

sports comportant des catégories de poids, la répartition des graisses oscille largement entre les périodes de perte et de reprise de poids (régime «yo-yo»). En conséquence, le pourcentage des graisses corporelles varie constamment, ce qui perturbe probablement le métabolisme des lipides au cours de la croissance.

\section{Micronutriments}

Des apports énergétiques inadéquats s'accompagnent généralement d'une très faible consommation de micronutriments (minéraux et vitamines). De fait, des études au cours desquelles de jeunes sportifs ont fait part de restrictions caloriques ont indiqué que les apports en micronutriments ne répondaient pas aux recommandations $[5,6,8,17,24,34$, $36,37]$. Des études ayant suivi les apports alimentaires chez des enfants et adolescents actifs ou de jeunes sportifs ont fréquemment identifié des carences en deux importants micronutriments: le calcium et le fer. Ces micronutriments sont capitaux non seulement pour une bonne santé, mais également pour l'optimisation des performances athlétiques. Le fer agit à titre de composante de diverses protéines, notamment des enzymes, l'hémoglobine et la myoglobine, cette dernière étant importante pour le transport de l'oxygène aux tissus dans l'ensemble de l'organisme à des fins de métabolisme. Une carence martiale induit une anémie et altère les performances. Le calcium est également un important micronutriment pour le développement de la masse osseuse, processus qui peut protéger contre une ostéoporose à l'âge adulte. Plus de $99 \%$ du calcium corporel total se trouvent dans les dents et les os. Le restant est présent dans le sang, le liquide extra-cellulaire, les muscles et d'autres tissus, où il joue un rôle dans la médiation de la vasoconstriction et de la vasodilatation, de la contraction musculaire, de la transmission nerveuse et de la sécrétion glandulaire.

La puberté accroît les besoins en fer et en calcium en raison d'une augmentation de la masse de l'hémoglobine, du dépôt tissulaire, de la poussée de croissance et de l'apparition des menstruations chez les jeunes filles. Les apports alimentaires en calcium et fer sont très bas dans les sports comportant des restrictions en énergie [38, 39], car les jeu- 
nes sportifs fuient les produits laitiers et la viande rouge, étant préoccupés par leur consommation de lipides ou parce qu'ils désirent un régime végétarien.

\section{Liquides et exercice physique}

Le statut d'hydratation avant la participation à des activités physiques ou à un sport est un important indice des performances et de l'endurance et peut limiter le risque de lésions chez l'adulte ainsi que chez l'enfant et l'adolescent. Un apport liquidien est recommandé avant et pendant l'activité physique et à la fin de celle-ci. Les liquides manquant à la fin de l'activité physique doivent être remplacés sur la base de la perte de poids.

Les informations scientifiques sur les sports à catégories de poids demeurent rares quant aux stratégies de déshydratation. Toutefois, d'un point de vue pratique, ces sportifs restreignent tout liquide avant la compétition (eau, soda, café, potage, etc.) et, de plus, en utilisant des sous-vêtements en matière plastique, ils suscitent généralement une sudation abondante au cours d'exercices physiques intensifs afin de stimuler une perte de poids par déperdition d'eau. D’autres stratégies telles que les saunas, les diurétiques et les vomissements sont habituellement adoptées afin de tenter de «faire le poids» $[5,28]$. Le bénéfice attendu d'une restriction liquidienne et d'une stratégie de déshydratation aiguë paraît être une perte de poids rapide permettant au sportif de concourir dans une catégorie spécifique. Cette stratégie, comme nous allons le voir, n'est toutefois pas sans conséquence.

\section{Conséquences potentielles pour la santé}

La difficulté est ici d'établir la relation de causalité entre les restrictions alimentaires et liquidiennes et les effets sur la «santé». En effet, l'interaction totale entre des facteurs génétiques et environnementaux (tels que la nutrition) complique la démonstration. Des études supplémentaires confortent toutefois l'explication des effets potentiellement négatifs sur la santé induits par les restrictions pondérales chez les sportifs enfants et/ou adolescents.

\section{Croissance et maturation}

Malgré la petite taille «normale» des gymnastes, gymnastes rythmiques et danseurs de haut niveau et les bénéfices manifestes pour la santé d'une activité physique au cours de la croissance, il est préoccupant d'envisager que les adeptes des «sports à critères esthétiques» participant à des programmes d'entraînement intensif puissent être exposés à un risque d'effets nocifs sur la croissance et la maturation. De fait, en l'absence d'une nutrition suffisante pour compenser l'accroissement du degré d'activité physique, les besoins énergétiques liés à l'entraînement peuvent entrer en compétition pour l'énergie disponible avec ceux des processus cellulaires sous-tendant la croissance et la maturation normale, particulièrement au cours de la poussée de croissance de l'adolescence [40].

Par exemple, la masse grasse est moins importante chez les gymnastes féminines que chez les témoins, et ces sportives sont également significativement moins grandes et plus légères pour leur âge, avec des différences plus importantes chez celles qui sont plus âgées et à un niveau plus élevé [41]. Le plus souvent, la maturation sexuelle est plus tardive chez les gymnastes des deux sexes que chez leurs jumeaux et/ou frères et sœurs moins actifs. Même si les études longitudinales de la croissance permettent une exploration plus approfondie que ce qu'autorisent des données transversales, elles ne démontrent toujours pas une relation de cause à effet [42]. Toutefois, la plupart d'entre elles indiquent un ralentissement de la croissance et un retard de la maturation chez les jeunes sportives participant à des programmes d'entraînement intensif, et de nombreuses données montrent la survenue d'une croissance de rattrapage quand la quantité totale d'entraînement diminue et/ou quand le régime alimentaire est suffisant [pour des détails, voir 42]. Des publications ont fréquemment indiqué des restrictions des apports énergétiques chez des adolescentes sportives de haut niveau. Ce mécanisme pourrait expliquer les effets nocifs sur la croissance et la maturation, car la poussée de croissance survenant lors de la puberté est sensible à des facteurs nutritionnels. Les mécanismes intervenant entre restriction alimentaire et ralentissement de la croissance et de la maturation chez les enfants et adolescents sont en partie dus à de moindres concentrations circulantes d'hormones agissant sur la croissance. En effet, nous savons que des restrictions caloriques et protéiques chroniques diminuent la concentration du facteur insulinique de croissance de type 1 (IGF-1) [43]. Comparativement à des témoins ou des nageurs et nageuses, les concentrations plasmatiques en IGF-1 sont plus basses chez les jeunes gymnastes féminines et sont associées à des apports énergétiques insuffisants, à une maturation retardée et à des perturbations de la croissance [44-46]. En revanche, ces mêmes concentrations ne sont pas réduites chez des gymnastes masculins en phase prépubertaire et pubertaire précoce comparativement à des témoins [47], mais il est bien connu que la restriction des apports alimentaires est toujours plus importante chez les filles.

\section{Composition corporelle}

La composition corporelle diffère en fonction du sport. Dans les disciplines sportives imposant une restriction pondérale, il existe une variabilité des mesures de la masse grasse et du poids corporel maigre relatifs entre les sports à 
critères esthétiques et les sports à catégories de poids, car des fluctuations pondérales apparaissent dans le second cas.

\section{Composition corporelle dans les sports à critères} esthétiques

La plupart des sportifs qui pratiquent des sports où l'apparence est un facteur important de succès sont très minces, car ils effectuent volontairement des restrictions alimentaires afin de maintenir un aspect physique prépubertaire avec un poids corporel bas et une masse grasse restreinte. En conséquence, une littérature étendue a montré que les gymnastes, les gymnastes rythmiques et les danseuses et danseurs en phase prépubertaire ou pubertaire précoce ou d'âge scolaire se caractérisaient par un pourcentage de graisse corporelle bas ou très bas [pour une analyse de la littérature, voir 48]. Paradoxalement, des études menées chez des sportifs ou des non sportifs ont démontré que la réponse adaptative humaine à une restriction énergétique était une réduction du métabolisme de repos, avec une augmentation éventuelle du stockage des graisses [49]. Il devient ainsi de plus en plus difficile à ces sportifs de conserver une masse grasse corporelle basse et, quand s'achève la période de compétition, une altération des tissus adipeux est habituellement observée, avec augmentation de la taille totale des adipocytes et/ou du nombre de ces cellules [15].

En règle générale, le déséquilibre énergétique créé par une activité physique très intense et associé à des restrictions alimentaires s'accompagne d'une densité minérale osseuse plus faible [49]. Toutefois, même si les apports calciques sont réduits chez les pratiquants de sports à critères esthétiques tels que la gymnastique classique ou rythmique, le patinage artistique et le ballet, ces jeunes sportifs des deux sexes présentent une densité minérale osseuse significativement plus élevée que d'autres groupes de sportifs ou de témoins non sportifs [48]. Des paramètres génétiques, et également les contraintes imposées aux os (sauts et réceptions au sol répétés), contribuent à une densité minérale osseuse plus élevée. De ce fait, quels que soient les apports nutritionnels, le contenu minéral osseux, et même la dimension des os, sont élevés chez les sportifs de haut niveau engagés dans des activités impliquant des impacts très importants.

\section{Composition corporelle dans les sports à catégories de poids}

Dans les sports comportant des catégories spécifiques de poids tels que la lutte, le judo, etc., les fluctuations pondérales sont rapides, fréquentes et importantes. De ce fait, la composition corporelle peut régulièrement se modifier. Les pertes de poids à court terme sont principalement composées d'eau, de graisses, de protéines et de glycogène. Quand le sportif perd du poids et stocke des graisses, la répartition des graisses corporelles peut se modifier. La contribution des stocks adipeux à la perte pondérale totale augmente substantiellement avec la durée des restrictions énergétiques. Toutefois, la plupart du temps, le jeune sportif s'engage rapidement dans un régime alimentaire et perd $\mathrm{du}$ poids sur de courtes périodes, ce qui aboutit à une perte significative de tissu maigre et d'eau $[5,15]$. La réduction du déficit énergétique et l'augmentation de la durée de la période de perte de poids devraient minimiser la perte de tissu maigre et augmenter la perte de tissu adipeux. Quand le sportif perd et regagne du poids, les stocks adipeux peuvent être perdus puis regagnés dans différents territoires de l'organisme [15]. Si des tissus adipeux disparaissent à un site et sont regagnés à un autre, leur distribution peut en être modifiée, même si la graisse totale demeure similaire. Les profils du gain pondéral chez les ex-pratiquants de sports à catégories de poids sont peu connus, mais, par la suite, le «régime yo-yo" pourrait induire une augmentation du rapport tour de taille/tour de hanches indiquant un risque d'affection cardiovasculaire, comme chez les sujets obèses.

\section{Efficience de l'alimentation}

L'efficience de l'alimentation peut être définie comme le rapport entre la modification du poids et les calories ingérées. Il représente un indice indiquant quelle doit être la consommation alimentaire d'une personne afin de maintenir un poids corporel ou une composition corporelle donné. Si l'efficience alimentaire augmente, les ingesta caloriques nécessaires au maintient d'un kilogramme de poids corporel diminuent. Sur la base de différentes études ayant examiné les apports nutritionnels et la composition corporelle chez de jeunes sportifs des deux sexes, Brownell et coll. [15] ont suggéré que l'efficience alimentaire pouvait être meilleure chez les sportifs dont le poids est bas ou fluctuant. Ce mécanisme correspond probablement à une adaptation physiologique facilitant le stockage de l'énergie à titre de processus vital. Toutefois, quand les apports caloriques augmentent, un gain pondéral pourrait rapidement apparaître. De façon anecdotique, certaines gymnastes se plaignent qu'il leur est de plus en plus difficile de maintenir leur poids bas, ce qui les engage dans une lutte chronique.

\section{Dysfonctions menstruelles et aptitude à la reproduction}

Dixon et coll. [50] ont suggéré que des activités physiques très intenses entraînaient une augmentation de la sécrétion d'endorphines et que ce phénomène, en association à des restrictions caloriques, induisait une régulation anormale de l'activité hypothalamique. La dysrégulation de l'hypothalamus déclenche la diminution de la fréquence de la sécrétion pulsatile de la gonadotrophine (hormone stimulant les folli- 
cules, hormone lutéinisante) et induit fréquemment un retard de la maturation pubertaire et, en conséquence, une oligoménorrhée ou une aménorrhée [8]. La régulation de l'axe hypothalamo-hypophysaire varie fortement d'un sport à l'autre. Il est bien connu que, chez les gymnastes féminines de haut niveau, l'âge de la ménarche tend à être plus tardif, et qu'une dysrégulation menstruelle (aménorrhée, oligoménorrhée, anovulation, déficit de phase lutéale) due à des altérations hormonales est présente et imputable au programme d'alimentation et d'entraînement [51]. Si une aménorrhée représente la forme la plus extrême de dysfonction menstruelle, d'autres peuvent également résulter en une réduction de la concentration des œstrogènes et affecter le métabolisme osseux et la fertilité. De ce fait, les filles physiquement actives et les adolescentes pratiquant des sports à critères esthétiques peuvent théoriquement présenter «la triade de la sportive», syndrome se composant de trois aspects: troubles del'alimentation, aménorrhée et ostéoporose (même si cette dernière est moins habituelle dans les activités impliquant des impacts élevés, comme précisé plus haut).

Une concentration nulle ou faible de la testostérone chez les sujets de sexe masculin peut être le parallèle de la dysfonction menstruelle chez les jeunes filles. Strauss et coll. [52] ont observé une testostéronémie basse chez des lutteurs, particulièrement quand le pourcentage de graisse corporelle diminuait au-dessous de 5\%. Ces concentrations sont revenues à la normale après la saison, moment où le poids a augmenté. De ce fait, un faible poids corporel, des tissus adipeux réduits, une sous-nutrition et/ou un entraînement intensif peuvent menacer les aptitudes à la reproduction des sportifs de sexe masculin.

\section{Statut martial et anémie}

Chez des sportifs entraînés, on enregistre fréquemment une hémoglobinémie basse (13-14 g/100 $\mathrm{ml}$ chez les hommes et $12 \mathrm{~g} / 100 \mathrm{ml}$ chez les femmes) ainsi qu'un hématocrite bas et une ferritinémie réduite $(<20 \mu \mathrm{g} / \mathrm{l})$. Ces paramètres définissent le concept "d'anémie du sport» [53]. La masse de l'hémoglobine augmente avec la puberté. A cette fin, et en raison d'un dépôt tissulaire, d'une poussée de croissance et de l'apparition des menstruations chez les jeunes filles, les besoins en fer sont plus élevés au cours de la période pubertaire [33]. Un faible statut martial peut être dû à une hémolyse mécanique, des saignements intestinaux, une hématurie, une hypersudation, une faible consommation de fer ou une absorption intestinale médiocre de celui-ci [53]. La diminution du transport des gaz du sang et de l'activité des enzymes musculaires qui en résulte altère les performances. Quelques études ont examiné le statut martial chez des adolescents sportifs, mais il semble que ces derniers, quand ils sont adultes, soient également sujets à une carence martiale non anémique $[54,55]$. D’autre part, des données indiquant une diminution des stocks de fer (ferritinémie basse) ont été décrites chez des gymnastes des deux sexes [38].

\section{Lésions}

De nombreuses études ont examiné les lésions survenant chez de jeunes gymnastes de haut niveau. Des études rétrospectives et prospectives ont montré que l'incidence des lésions était élevée chez les gymnastes féminines et positivement associée au degré de performance. Des lésions aiguës (par exemple fractures de contrainte et entorses ligamentaires) affectant le pied et les orteils sont fréquentes, il en est de même des traumatismes de la cheville [48]. Certaines de ces lésions aiguës pourraient être dues à des restrictions alimentaires, car il est bien connu qu'une œstrogénémie basse et des apports protéiques et calciques insuffisants, associés à une ménarche tardive, peuvent aboutir à une augmentation de l'incidence des fractures de contrainte [56].

Au cours des stratégies de diminution pondérale dans les sports à catégories de poids, il est habituel d'observer des lésions musculosquelettiques dues à une privation liquidienne avant la compétition [35]. Une possibilité de lésion thermique est également présente si la compétition est effectuée dans un environnement très chaud.

\section{Effets de la déshydratation}

Une réduction pondérale sévère pourrait effectivement altérer les fonctions physiologiques. Par exemple, une déshydratation pourrait nuire aux reins en réduisant le flux et la filtration plasmatiques. De jeunes lutteurs ont également fait part d'accès d'irritabilité, de constipation et du fait de «se sentir à plat» [37]. Les effets d'une perte pondérale rapide et d'une déshydratation sur les performances physiologiques sont bien documentés [35]. Les conséquences de ces pratiques pourraient être les suivantes: réduction de la force musculaire et de la capacité de puissance aérobie, augmentation de la fréquence cardiaque au repos et submaximale, diminution du volume d'éjection cardiaque résultant en une réduction de l'aptitude à effectuer un travail à un taux constant, plus basse consommation d'oxygène, altération des processus de thermorégulation, plus bas volume plasmatique et sanguin, déplétion du glycogène musculaire et peut-être hépatique, difficulté de l'homéostasie du glucose, altération du statut hormonal, réduction de la fonction immunitaire, etc. [35]. De plus, une étude in vitro a suggéré qu'une déshydratation cellulaire pouvait aboutir à l'instauration d'un catabolisme des protéines et du glycogène comme moyen d'adapter l'osmolarité cellulaire en réponse à une diminution du volume liquidien intracellulaire [57]. 


\section{Troubles de l'alimentation}

Les troubles de l'alimentation peuvent être définis comme la consommation ou non d'aliments en réponse à un stimulus externe plutôt qu'interne. Les troubles de l'alimentation dans la population sportive féminine font l'objet d'une attention croissante. Les sportifs pratiquant des sports qui nécessitent une minceur et/ou un poids corporel spécifique sont plus sujets aux troubles alimentaires que les autres [58]. Ainsi, les compétiteurs de gymnastique rythmique, les membres de compagnies de ballet et les patineurs artistiques, par exemple, sont suspects de favoriser une réduction pondérale non physiologique pouvant évoluer en une anorexie mentale et/ou en une boulimie nerveuse manifestes [58]. La pression colossale que subissent les jeunes sportifs pour atteindre et conserver un poids corporel idéal peut en être l'explication. De ce fait, les pratiquants des sports où la minceur est nécessaire font part d'une insatisfaction plus importante de leur corps, d'une moindre estime de soi et de régimes alimentaires plus prolongés que les autres sportifs. L'inaptitude à contrôler le poids et les formes du corps peut aboutir à des sentiments de frustration et de culpabilité, voire de désespoir, qui peuvent induire des perturbations sociales et affectives.

\section{Morbidité et mortalité}

Les pratiques alimentaires suivies par certains sportifs peuvent accroître le risque de maladies aiguës ou chroniques. Outre la qualité spécifique de l'alimentation (graisses saturées, sucres simples, sodium, etc.), les variations du poids corporel pourraient également influencer la santé. Il est bien connu qu'une restriction calorique chronique réduit le risque de certains cancers [59] et diminue les coronaropathies, l'hypertension et le diabète. Toutefois, les sportifs dont le poids corporel est faible et qui souffrent de troubles alimentaires peuvent être victimes d'importantes carences nutritionnelles (par exemple vitamines, minéraux, acides aminés essentiels, acides gras essentiels), ce qui peut altérer des fonctions métaboliques et organiques. Par exemple, les patientes atteintes d'anorexie mentale présentent une dispersion de QT plus importante que les femmes constitutionnellement minces et de poids normal, en raison d'une kaliémie très basse (la dispersion de QT a été définie comme la différence entre les intervalles QT les plus longs et les plus courts sur un ECG à 12 dérivations) [60]. De même, des épisodes répétés de perte et de reprise de poids peuvent exercer différents effets négatifs sur la santé. En effet, une perte de poids est associée à une diminution de la pression artérielle, et un gain pondéral à une augmentation de celle-ci. La période d'augmentation étant plus importante que la période de diminution, la pression artérielle peut être affectée. De plus, différentes études ont suggéré qu'une ischémie rénale pouvait résulter de variations cycliques du poids chez des lutteurs, ce qui pourrait les prédisposer à une hypertension ultérieure [15]

\section{Conclusion}

Comme la présente analyse l'a montré, les conséquences des restrictions pondérales imposées par le sport sur la santé de l'enfant et de l'adolescent sont nombreuses, et certaines sont irréversibles. Afin d'éviter ces effets dramatiques, nous recommandons un suivi attentif du statut nutritionnel des jeunes sportifs très actifs, quels que soient leur âge, leur discipline sportive et leur sexe, particulièrement ceux qui pratiquent des sports caractérisés par une limitation des apports nutritionnels (sports à critères esthétiques) ou par une consommation alimentaire irrégulière (sports reposant sur des catégories de poids). Une approche psychologique et une éducation nutritionnelle des sportifs, des entraîneurs, des parents, etc., devraient compléter les conseils diététiques donnés par un diététicien ou un nutritionniste du sport.

\section{Références}

1 DRI: Dietary Reference Intakes for Energy, Carbohydrate, Fiber, Fat, Fatty Acids, Cholesterol, Protein, and Amino Acids (Macronutrients). Washington, National Academy Press, 2002.

2 Tveit-Milligan P, Spindler AA, Nichols JE: Genes and gymnastics: a case study of triplets. Sports Med Training Rehab 1993;4:47-52.

$\checkmark 3$ Lindholm C, Hagenfeldt K, Ringertz BM: Pubertal development in elite juvenile gymnasts. Effects of physical training. Acta Obstet Gynecol Scand 1994;73:269-273.
4 Steen SN, Brownell KD: Patterns of weight loss and regain in wrestlers: has the tradition changed? Med Sci Sports Exerc 1990;22:762768.

5 Boisseau N, Vera-Perez S, Poortmans JR: Food and fluid intake in adolescent female judo athletes before competition. Pediatr Exerc Sci 2005; 17:62-71.

6 Oppliger RA, Steen SA, Scott JR: Weight loss practices of college wrestlers. Int J Sport Nutr Exerc Metab 2003;13:29-46.

7 Steen SN, Oppliger RA, Brownell KD: Metabolic effects of repeated weight loss and regain in adolescent wrestlers. JAMA 1988;260:4750
>8 Weimann E, Witzel C, Schwidergall S, Bohles HJ: Peripubertal perturbations in elite gymnasts caused by sport specific training regimes and inadequate nutritional intake. Int J Sports Med 2000;21:210-215.

9 Theintz GE, Howald H, Allemann Y, Sizonenko PC: Growth and pubertal development of young female gymnasts and swimmers: a correlation with parental data. Int J Sports Med 1989;10:87-91.

10 Schwidergall S, Weimann E, Witzel C, Molenkamp G, Brehl S, Bohles H: Nutritional behaviour of female and male high performance gymnasts. Wien Med Wochenschr 1988;148: 243-244. 
11 Lindholm C, Hagenfeldt K, Hagman U: A nutrition study in juvenile elite gymnasts. Acta Paediatr 1995;84:273-277.

12 Kiningham RB, Gorenflo DW: Weight loss methods of high school wrestlers. Med Sci Sports Exerc 2001;33:810-813.

13 Gurd B, Klentrou P: Physical and pubertal development in young male gymnasts. J Appl Physiol 2003;95:1011-1015.

14 Georgopoulos N, Markou K, Theodoropoulou A, Paraskevopoulou P, Varaki L: Growth and pubertal development in elite female rhythmic gymnasts. J Clin Endocrinol Metab 1999;84:4525-4530.

15 Brownell KD, Steen SN, Wilmore JH: Weight regulation practices in athletes: analysis of metabolic and health effects. Med Sci Sports Exerc 1987;19:546-556.

16 Baxter-Jones AD, Helms P, Maffulli N, BainesPreece JC, Preece M: Growth and development of male gymnasts, swimmers, soccer and tennis players: a longitudinal study. Ann Hum Biol 1995;22:381-394.

-17 Boisseau N, Persaud C, Jackson AA, Poortmans JR: Training does not affect protein turnover in pre- and early pubertal female gymnasts. Eur J Appl Physiol 2005;94:262267.

18 Fogelholm GM, Kukkonen-Harjula TK, Taipale SA, Sievänen HT, Oja P, Vuori IM: Resting metabolic rate and energy intake in female gymnasts, figure-skaters and soccer players. Int J Sports Med 1995; 16:551-556.

19 Bass S, Bradney M, Pearce G, Hendrich E, Inge $\mathrm{K}$ : Short stature and delayed puberty in gymnasts: influence of selection bias on leg length and the duration of training on trunk length. J Pediatr 2000;136:149-155.

20 Zonderland ML, Claessens AL, Lefevre J, Philippaerts R, Thomis M: Delayed growth and decreased energy intake in female gymnasts; in Armstrong N, Kirby B, Welsman J (eds): Children and Exercise XIX: Promoting Health and Well-Being: Proceedings of the XIXth International Symposium of the European Group of Pediatric Work Physiology. London, E\&FN Spon, 1997, pp 553-556.

21 Poortmans JR: Protein metabolism; in Poortmans JR (ed): Principles of Exercise Biochemistry. Basel, Karger, 2004, pp 227-278.

22 FAO/WHO: Joint Expert Committee: Energy and Protein Requirements. Geneva, World Health Organization, 1986.

23 Martin A: Apports nutritionnels conseillés pour la population française. Paris, Tec \& Doc, 2001.

-24 Cupisti A, D’Alessandro S, Castrogiovanni S, Barale E, Morelli E: Nutrition survey in elite rhythmic gymnasts. J Sports Med Phys Fit 2000;40:350-355.

25 Weimann E, Blum WF, Witzel C, Schwidergall S, Bohles HJ: Hypoleptinemia in female and male elite gymnasts. Eur J Clin Invest 1999;29:853-860.

26 Fogelholm GM, Kukkonen-Harjula TK, Taipale SA, Sievanen HT, Oja P, Vuori IM: Resting metabolic rate and energy intake in female gymnasts, figure-skaters and soccer players. Int J Sports Med 1995;16:551-556.
27 Benson J, Gillien DM, Bourdet K, Loosli AR: Inadequate nutrition and chronic calorie restriction in adolescent ballerinas. Phys Sportmed 1985;13:79-90.

28 Horswill CA, Park SH, Roemmich JN: Changes in the protein nutritional status of adolescent wrestlers. Med Sci Sports Exerc 1990;22: 599-604.

29 Friedlander AL, Braun B, Pollack M, MacDonald JR, Fulco CS: Three weeks of caloric restriction alters protein metabolism in normal-weight, young men. Am J Physiol Endocrinol Metab 2005;289:E446-E455.

30 Coyle EF, Coyle E: Rating carbohydrates that speed recovery from training. Phys Sportsmed 1993;21:111-123.

31 Costill DL, Sherman WM, Fink WJ, Maresh C, Witten M, Miller JM: The role of dietary carbohydrate in muscle glycogen resynthesis after strenuous running. Am J Clin Nutr 1981; 34:1831-1836.

32 Boisseau N, Delamarche P: Metabolic and hormonal responses to exercise in children and adolescents. Sports Med 2000;30:405422.

33 ANC: Apports nutritionnels recommandés (ANC) pour la population française. Paris, Tec \& Doc, 2001.

34 Delistraty DA, Reisman EJ, Snipes MG: A physiological and nutritional profile of young female figure skaters. J Sports Med Phys Fit 1992;32:149-155.

35 Oppliger RA, Case HS, Horswill CA, Landry GL, Shelter AC: American College of Sports Medicine position stand. Weight loss in wrestlers. Med Sci Sports Exerc 1996;28:ix-xii.

36 Moffatt RJ: Dietary status of elite female high school gymnasts: inadequacy of vitamin and mineral intake. J Am Diet Assoc 1984;84: 1361-1363.

37 Freischlag J: Weight loss, body composition, and health of high school wrestlers. Phys Sportmed 1984;12:121-126.

38 Constantini NW, Eliakim A, Zigel L, Yaaron M, Falk B: Iron status of highly active adolescents: evidence of depleted iron stores in gymnasts. Int J Sport Nutr Exerc Metab 2000;10: 62-70.

-39 Kirchner EM, Lewis RD, O’Connor PJ: Bone mineral density and dietary intake of female college gymnasts. Med Sci Sports Exerc 1995; 27:543-549.

$\checkmark 40$ Borer KT: The effects of exercise on growth. Sports Med 1995;20:375-397.

41 Caine D, Lewis R, O’Connor P, Howe W, Bass $S$ : Does gymnastics training inhibit growth of females? Clin J Sport Med 2001;11:260-270.

42 Caine D, Bass S, Daly R: Does elite competition inhibit growth and delay maturation in some gymnasts? Quite possibly. Pediatr Exerc Sci 2003; 15:360-372.

43 Smith WJ, Underwood LE, Clemmons DR: Effects of caloric or protein restriction on insulin-like growth factor-I (IGF-I) and IGFbinding proteins in children and adults. J Clin Endocrinol Metab 1995;80:443-449.

44 Theintz GE: Endocrine adaptation to intensive physical training during growth. Clin Endocrinol 1194;41:267-272.
45 Jahreis G, Kauf E, Frohner G, Schmidt HE: Influence of intensive exercise on insulin-like growth factor I, thyroid and steroid hormones in female gymnasts. Growth Regul 1991;1:9599.

46 Bass S, Pearce G, Bradney M, Hendrich E, Delmas PD: Exercise before puberty may confer residual benefits in bone density in adulthood: studies in active prepubertal and retired female gymnasts. J Bone Miner Res 1998;13: 500-507.

47 Daly RM, Rich PA, Klein R, Bass SL: Short stature in competitive prepubertal and early pubertal male gymnasts: the result of selection bias or intense training? J Pediatr 2000; 137:510-516.

48 O'Connor PJ, Lewis RD, Boyd A: Health concerns of artistic women gymnasts. Sports Med 1996:21:321-325.

49 Deutz RC, Benardot D, Martin DE, Cody MM: Relationship between energy deficits and body composition in elite female gymnasts and runners. Med Sci Sports Exerc 2000;32: 659-668.

50 Dixon G, Eurman P, Stern BE, Schwartz B, Rebar RW: Hypothalamic function in amenorrheic runners. Fertil Steril 1984;42:377-383.

51 Manore MM: Dietary recommendations and athletic menstrual dysfunction. Sports Med 2002;32:887-901.

52 Strauss RH, Lanese RR, Malarkey WB: Weight loss in amateur wrestlers and its effect on serum testosterone levels. JAMA 1985;254: 3337-3338.

53 Chatard JC, Mujika I, Guy C, Lacour JR: Anaemia and iron deficiency in athletes. Practical recommendations for treatment. Sports Med 1999;27:229-240.

54 Rowland TW: Iron deficiency in the young athlete. Pediatr Clin North Am 1990;37:11531163.

55 Rowland TW, Stagg L, Kelleher JF: Iron deficiency in adolescent girls. Are athletes at increased risk? J Adolesc Health 1991;12: $22-25$.

56 Benson JE, Geiger CJ, Eiserman PA, Wardlaw GM: Relationship between nutrient intake, body mass index, menstrual function, and ballet injury. J Am Diet Assoc 1989;89:5863.

57 Petrie HJ, Stover EA, Horswill CA: Nutritional concerns for the child and adolescent competitor. Nutrition 2004;20:620-631.

58 Sundgot-Borgen J: Eating disorders, energy intake, training volume, and menstrual function in high-level modern rhythmic gymnasts. Int J Sport Nutr 1996;6:100-109.

59 Kritchevsky D, Weber MM, Buck CL, Klurfeld DM: Calories, fat and cancer. Lipids 1986; 21:272-274.

60 Franzoni F, Mataloni E, Femia R, Galetta F: Effect of oral potassium supplementation on QT dispersion in anorexia nervosa. Acta Paediatr 2002;91:653-656. 